\title{
Drug resistance and minimal residual disease in multiple myeloma
}

\author{
Alessandro Gozzetti, Sara Ciofini, Anna Sicuranza, Paola Pacelli, Donatella Raspadori, Emanuele Cencini, \\ Dania Tocci, Monica Bocchia
}

Hematology, University of Siena, Azienda Ospedaliera Universitaria Senese, Siena 53100, Italy.

Correspondence to: Prof. Alessandro Gozzetti, Hematology, University of Siena, Policlinico Le Scotte, Viale Bracci 16, Siena 53100, Italy. E-mail: gozzetti@unisi.it

\begin{abstract}
How to cite this article: Gozzetti A, Ciofini S, Sicuranza A, Pacelli P, Raspadori D, Cencini E, Tocci D, Bocchia M. Drug resistance and minimal residual disease in multiple myeloma. Cancer Drug Resist 2022;5:171-83. https://dx.doi.org/10.20517/cdr.2021.116
\end{abstract}

Received: 1 Nov 2021 First Decision: 5 Jan 2022 Revised: 17 Jan 2022 Accepted: 29 Jan 2022 Published: 16 Feb 2022

Academic Editors: Godefridus J. Peters, Fatih M. Uckun Copy Editor: Yue-Yue Zhang Production Editor: Yue-Yue Zhang

\begin{abstract}
Great progress has been made in improving survival in multiple myeloma (MM) patients over the last 30 years. New drugs have been introduced and complete responses are frequently seen. However, the majority of MM patients do experience a relapse at a variable time after treatment, and ultimately the disease becomes drugresistant following therapies. Recently, minimal residual disease (MRD) detection has been introduced in clinical trials utilizing novel therapeutic agents to measure the depth of response. MRD can be considered as a surrogate for both progression-free and overall survival. In this perspective, the persistence of a residual therapy-resistant myeloma plasma cell clone can be associated with inferior survivals. The present review gives an overview of drug resistance in $M M$, i.e., mutation of $\beta 5$ subunit of the proteasome; upregulation of pumps of efflux; heat shock protein induction for proteasome inhibitors; downregulation of CRBN expression; deregulation of IRF4 expression; mutation of CRBN, IKZF1, and IKZF3 for immunomodulatory drugs and decreased target expression; complement protein increase; $\mathrm{SBCMA}$ increase; and BCMA down expression for monoclonal antibodies. Multicolor flow cytometry, or next-generation flow, and next-generation sequencing are currently the techniques available to measure MRD with sensitivity at $10^{-5}$. Sustained MRD negativity is related to prolonged survival, and it is evaluated in all recent clinical trials as a surrogate of drug efficacy.
\end{abstract}

Keywords: Multiple myeloma, minimal residual disease, drug resistance, therapy, next-generation flow cytometry 


\section{INTRODUCTION}

Multiple myeloma ( $\mathrm{MM}$ ) represents the second most frequent hematological malignancy, and significant survival improvements in the years have been $\operatorname{see}^{[1-3]}$. Survival has ameliorated thanks to the availability of novel classes of drugs such as proteasome inhibitors (PIs: bortezomib, and carfilzomib), immunomodulatory drugs (IMIDs: thalidomide, lenalidomide, and pomalidomide), monoclonal antibodies (moAb: daratumumab, elotuzumab, isatuximab, and belantamab mafodotin). These new drugs are utilized alone or combined as triplets or quadruplets in the treatment of relapsed/refractory patients and thereafter at diagnosis with good results also in extramedullary $\mathrm{MM}^{[4-17]}$. $\mathrm{MM}$ has important clinical heterogeneity and complex genetic abnormalities ${ }^{[18]}$. Cytogenetic analysis and fluorescence in situ hybridization can help to distinguish different categories and risk-stratify $M M$ patients $^{[19]}$. In particular, $t(4 ; 14), t(14 ; 20)$, gain of chromosome 1q, and deletion $17 \mathrm{p}$ give a poorer outcome, while $\mathrm{t}(11 ; 14)$ and $\mathrm{t}(14 ; 16)$ seem to have an intermediate prognosis ${ }^{[20-22]}$. In this view, relapses are seen in the majority of patients, and some of them still have a dismal prognosis. However, while cytogenetic analysis can help to stratify prognosis, it does not fully explain initial MM drug resistance (DR). Two main factors seem to emerge: (1) acquired drug resistance; and (2) sub-clonal heterogeneity ${ }^{[2,24]}$. Mechanisms of acquired drug resistance to the main classes of new drugs available in $\mathrm{MM}$ have been extensively reviewed recently ${ }^{[22]}$. A novel method to detect minimal residual disease has been developed recently ${ }^{[23-35]}$, i.e., MRD detected by next-generation flow (NGF) or next-generation sequencing (NGS), which have been reported as important tools by the International Myeloma Working Group (IMWG) in the recent response guidelines ${ }^{[36]}$. MRD is an important surrogate for survival, as well as progression-free (PFS) and overall survival (OS $)^{[37]}$. In particular, sustained MRD negativity confirmed at one year is of great importance to predict clinical outcome of MM patients. MRD can also detect sub-clones that acquire higher genomic instability and can ultimately drive resistance ${ }^{[38]}$. This review summarizes the main DR cell-inherent/intrinsic and extrinsic mechanisms to novel drugs in MM and will focus on recent developments regarding MRD as a tool to predict PFS and OS in clinical trials.

\section{Drug resistance in MM}

Drug resistance is the leading cause of a relapsed/refractory disease, and it can ultimately decrease survival. Many novel drugs are now available in MM with many used at diagnosis in triplet or quadruplet. MM patients can develop DR after a few cycles of therapy in a variable manner. The main mechanisms of resistance to chemotherapeutic drugs in MM can involve a drug-efflux pump, such as P-glycoprotein, or other mechanisms that inhibit the drug to enter the cell. Moreover, enzymatic inactivation of the drug or adhesion to bone marrow stromal cells, such as fibroblasts and immune cells including macrophages, in the microenvironment can be mentioned. To develop strategies for the future treatment of DR-MM patients, the main mechanisms of resistance to the different classes of drugs, namely proteasome inhibitors, immunomodulatory drugs, and monoclonal antibodies, have to be elucidated. The principal mechanisms of resistance to novel drugs and resistance escape are reported in Table 1.

\section{Drug resistance to proteasome inhibitors}

The PIs used in recent years in clinical practice are bortezomib (Bor), carfilzomib (Car), and ixazomib $(\mathrm{Ixa})^{[39,40]}$. Bor is approved for use at diagnosis in both transplant and non-transplant eligible MM patients, while Car and Ixa are used in the relapsed and refractory treatment setting. The structure of the proteasome was first described as a hollow single-cylinder protein. The proteasome functions as an ATP-dependent organelle in which almost $90 \%$ of intracellular proteins are degraded following tagging by the polyubiquitin chain. Three subunits of the proteasome are recognized as the site of degradation in the $20 \mathrm{~S}$ particle: $\beta 1$ (caspase), $\beta 2$ (trypsin), and $\beta 5$ (chymotrypsin). The final result of proteasome inhibition is MM plasma cell death or apoptosis via protein accumulation ${ }^{[41]}$. Other mechanisms have been reported: p53 activation, inhibition of nuclear factor- $\mathrm{\kappa B}(\mathrm{NF}-\mathrm{\kappa} \mathrm{B})$ activity, activation of c-Jun N-terminal kinase, and stabilization of cell cycle inhibitors. PI can bind the $\beta 5$ subunit reversibly (Bor and Ixa) or irreversibly (Car). Resistance to 


\section{Table 1. Principal mechanisms of drug resistance and resistance escape}

\begin{tabular}{|c|c|c|c|c|c|}
\hline C & D & M of $A$ & DR & Resistance escape & Ref. \\
\hline moAb & $\begin{array}{l}\text { Dara } \\
\text { Isa } \\
\text { Elo } \\
\text { Bela }\end{array}$ & $\begin{array}{l}\text { ADCC, CDC, macrophage-mediated phagocytosis, apoptosis via Fc-mediated } \\
\text { crosslinking stimulatory effects on NK cells (for anti CD38 moAb), direct cytotoxicity }\end{array}$ & $\begin{array}{l}\text { Decrease target expression, complement } \\
\text { protein increase, sBCMA increase, BCMA } \\
\text { down expression }\end{array}$ & $\begin{array}{l}\text { Change drug class, upregulation of CD38 using } \\
\text { ATRA }\end{array}$ & [61-67] \\
\hline IMIDs & $\begin{array}{l}\text { Tha } \\
\text { Lena } \\
\text { Poma }\end{array}$ & $\begin{array}{l}\text { BM microenvironment targeting; degradation of IKZF1 and IKZF3 via CRBN-dependent } \\
\text { ubiquitination; IRF4 and MYC downregulation; triggering caspase } 8 / 9 \text {-mediated } \\
\text { apoptosis; immune modulation; anti-angiogenic activity }\end{array}$ & $\begin{array}{l}\text { Downregulation of CRBN expression; } \\
\text { deregulation of IRF4 expression } \\
\text { Mutation of CRBN and IKZFI and IKZF3 }\end{array}$ & $\begin{array}{l}\text { Change drug class, next-generation CRBN E3 } \\
\text { ligase modulators (iberdomide) }\end{array}$ & {$[58,59]$} \\
\hline Pls & $\begin{array}{l}\text { Bort } \\
\text { Car } \\
\text { Ixa }\end{array}$ & $\begin{array}{l}\text { Inhibition of activity of the } 20 \mathrm{~S} \text { proteasome; inhibition of NF-kB activity; induction of } \\
\text { apoptosis by activation of caspase } 8 / 9 \text { and p53; adhesion molecules downregulation }\end{array}$ & $\begin{array}{l}\text { Mutation of } \beta 5 \text { subunit, upregulation of } \\
\text { pumps of efflux, HSP induction }\end{array}$ & $\begin{array}{l}\text { Change drug class, pan proteasome inhibitor } \\
\text { (marizomib), hydroxychloroquine, pan HDAC } \\
\text { inhibitor }\end{array}$ & {$[46,48]$} \\
\hline
\end{tabular}

C: Class of drug; D: drug name; M of A: mechanisms of action; DR: drug resistance; moAb: monoclonal antibody; IMIDs: immunomodulatory drug; PI: proteasome inhibitors; Dara: daratumumab; Isa: isatuximab; Elo: elotuzumab; Bela: belantamab mafodotin; Tha: thalidomide; Lena: lenalidomide; Poma: pomalidomide; Bor: bortezomib; Car: carfilzomib; Ixa: ixazomib; ADCC: antibody-dependent cellular cytotoxicity; CDC: complement-dependent cytotoxicity; BCMA: b cell maturation antigen; ATRA: all trans retinoic acid; IKZFT: Ikaros; IKZF3: Aiolos; BM: bone marrow; CRBN: Cereblon; IRF4: interferon regulatory factor 4; NF-KB: nuclear factor-kB; HDAC: histone deacetylase; HSP: heat shock protein.

PIs is related to mutations in the $\beta 5$ subunit gene (PSMB5) at both diagnosis and relapse ${ }^{[42,43]}$. The presence of mutations at other subunits, namely PSMA1, $P S M B 8$, and $P S M B 9$, has been described, but they have not been found to be related to resistance yet and thus need to be confirmed. Another protein important for DR seems to be the X-box protein 1 that acts in the proteostasis in the MM plasma cell. Downregulation of this pathway has been reported in PIDR cells ${ }^{[44]}$. Decreased drug accumulation is another mechanism of DR in MM. In particular, the multidrug resistance protein P-glycoprotein (P-gp) has been well studied in the past ${ }^{[45]}$. P-gp works by implementing drug efflux from the MM plasma cell, thus reducing drug activity. Both Car and Bor have been reported to be P-gp substrates ${ }^{[46-48]}$. The mechanisms of resistance escape are reported in Table $1^{[4,50]}$. The results of preclinical studies indicate that inhibition of various heat shock proteins (HSPs), e.g., HSP90, can increase the efficacy of PIs ${ }^{[51]}$. HSPs are induced by the transcriptional blockade of protein degradation, which can contribute to drug resistance. The results from early phase I trials combining HSP9o inhibitors with PIs have identified safe doses for both drugs. In a preclinical study using bor-resistant MM cell lines, the blockade of IGF-1 downstream effectors re-sensitized cell lines to bortezomib. Furthermore, the IGF$1 \mathrm{R}$ inhibitor OSI-906 induced more apoptosis than Bor alone, both in vitro and in vivo ${ }^{[52]}$.

Moreover, investigators used a mouse MM model to study gene-expression signatures (GES) related to Bor resistance. GES related to resistance included nuclear factor (erythroid-derived 2)-like 2 (NFE2L2), highly expressed as part of an antioxidant-response pathway ${ }^{[33]}$. Thus, MM cells with elevated antioxidant capacity before treatment might be resistant to bortezomib. 


\section{Drug resistance to immunomodulatory drugs}

The IMIDs currently used in clinical practice are thalidomide, lenalidomide, and pomalidomide (Thal, Len, and Pom, respectively). All three agents were initially used in the relapsed and refractory setting, but Len and Thal are now also approved for use at diagnosis ${ }^{[54]}$. IMIDs have antiproliferative properties and direct pro-apoptotic effects. Other mechanisms exhibited are anti-angiogenic and immunomodulatory effects, by activating NK and T cells. The E3 ligase protein Cereblon $(C R B N)$ is targeted by IMIDs, in particular in complex with CRL4, ultimately leading to degradation of the Aiolos and Ikaros proteins and IRF4 reduction ${ }^{[55-57]}$. This mechanism of action is the principal target of DR, mainly because of occurring mutations or gene expression modifications in this complex. It has been demonstrated that CRBN mutations appear after a relapsed/refractory disease occurs, probably due to clonal selection after long IMID therapy ${ }^{[58]}$. In addition, IRF4 has been investigated as a potential downstream target for DR in Waldenstrom macroglobulinemia after therapy with Len and Pom $^{[59]}$. Methylation of CRBN has also been recently reported $^{[60]}$.

\section{Drug resistance to monoclonal antibodies}

Monoclonal antibodies directed at antigens present on the surface of plasma cells have recently entered the therapeutic armamentarium against MM. The approved moAb in clinical practice are daratumumab, elotuzumab, isatuximab, and belantamab mafodotin. Thse drugs were all tested as monotherapy first, and have since been proven to be more efficacious when combined with other agents in triplet or quadruplet $^{[61-65]}$.

Even though daratumumab, an IgG 1 anti-CD38 moAb, has been found to be very efficacious, resistance can be observed during treatment. Although CD38 mutations are not described as potential mechanism of $\mathrm{DR}$, it has been reported that low $\mathrm{CD} 38$ expression could be a cause of initial resistance to therapy. Moreover, CD38 expression reduction during treatment has been described as a major mechanism and occurs via the action of sheddases ${ }^{[66]}$. Another occurring event observed is upregulation of CD55 and CD59 complement inhibitors ${ }^{\left[{ }^{[6]}\right]}$. For other moAb, the DR mechanism is not currently known, although it is logical to think that, as per daratumumab, the loss of the target antigen is likely a major mechanism of resistance ${ }^{[68-72]}$.

\section{Extrinsic mechanisms of drug resistance}

The bone marrow microenvironment, including osteoblasts, osteoclasts, mesenchymal stem cells (MSC), and tumor-associated macrophages (TAM), is highly connected with MM progression and drug resistance [Figure 1]. In MM in the "osteoblastic niche", macrophages are differentiated into osteoclasts by the release of osteoclasts activating factors (i.e., IL-6, IL-1- $\alpha$, TNF- $\alpha$, TNF- $\beta$, and IL-11) and promote bone resorption. Osteoprotegerin represents an antiapoptotic MM factor by binding to TNF-related apoptosis-inducing ligand. Stromal cells can give drug resistance by TGF- $\beta$ inhibition of osteoblasts differentiation. Stromal cells in the microenvironment have been shown to secrete several cytokines that regulate the antiapoptotic members of the Bcl-2 family ( $\mathrm{Mcl}-1, \mathrm{Bcl}-\mathrm{xl}$, and Bcl2) via IL-6 signaling ${ }^{[73]}$. A "vascular niche" is formed by endothelial cells (EC), MSC, and TAM and can protect MM plasma cells from cytotoxic drugs. In particular, EC can express an aberrant active phenotype (VEGFR-2 and FGFR-3) that can help to prevent MM apoptosis, favoring PC migration into the bloodstream and dissemination ${ }^{[7]}$. MSC can contribute to bortezomib resistance in $\mathrm{MM}$ via Bcl2 increased expression and enhanced $\mathrm{NF}-\mathrm{\kappa B}$ activity through cell-cell $\operatorname{contact}^{[74]}$.

TAM have a basic role in MM pathogenesis, since they promote plasma cells proliferation, homing, and angiogenesis, supporting MM immune evasion and progression ${ }^{[75]}$. 


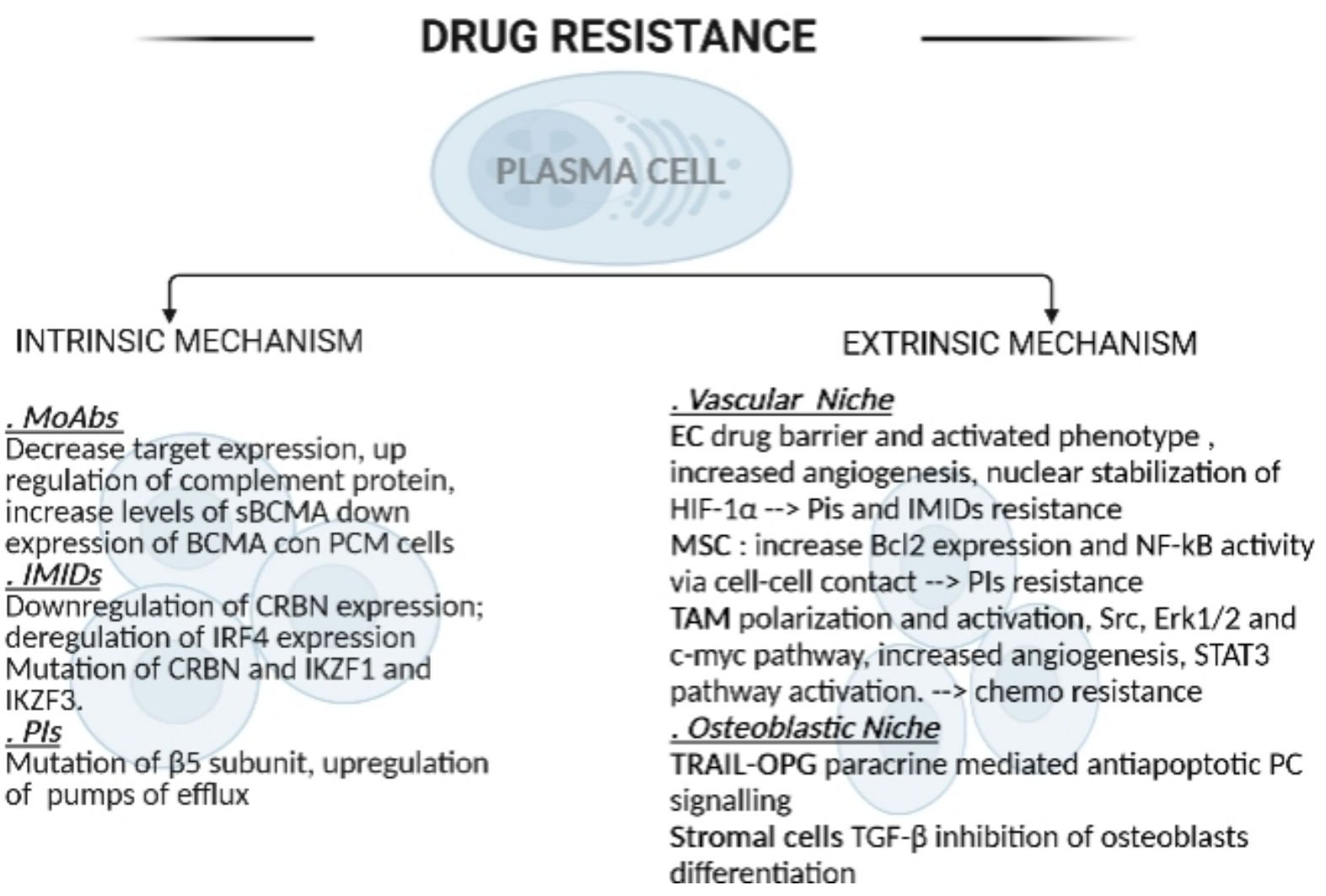

Figure 1. Drug resistance intrinsic and extrinsic mechanisms. EC: Endothelial cells; MSC: mesenchymal stem cells; TAM: tumorassociated macrophages.

MM plasma cells in vitro could upregulate CD206 expression and favor an M2 TAM polarization of cocultured macrophages ${ }^{[76]}$. In a preclinical model, CD68-positive TAM were shown to inhibit druginduced apoptosis of tumor cells by caspase 3 and poly-ADP ribose polymerase cleavage ${ }^{[77,78]}$. Moreover, intracellular adhesion molecule-1 (ICAM-1) and P-selectin glycoprotein ligand-1 (PSGL-1) on the MM cells surface could activate TAM and favor TAM-induced chemoresistance through the $S R C, E R K 1 / 2$, and $C$ $M Y C$ pathway ${ }^{[79]}$. VEGF production by M2 TAM was demonstrated during progression from MGUS to $\mathrm{MM}$, with increased angiogenic switch ${ }^{[00,81]}$.

An interesting study reported a possible correlation between the pro-tumor effect of TAM and the Stat3 pathway activation in $5 \mathrm{~T} 33 \mathrm{MM}$ cells. Interestingly, an ATP-competitive Janus kinase (JAK)2 inhibitor, the so-called AZD1480, could restore the sensitivity of MM cells to bortezomib ${ }^{[82,83]}$.

Clinical studies confirmed CD68/CD163 double-positive M2 TAM were associated with increased microvessel density and reduced survival, independently of the MM stage ${ }^{[84-86]}$. In this field, high IHC CD163-positive M2 TAM expression at diagnosis was associated with lower complete response (CR) rate and reduced PFS and OS in $198 \mathrm{MM}$ patients receiving bortezomib-based regimen ${ }^{[84]}$. Interestingly, an elevated level of soluble M2 TAM markers CD163 and CD206 was associated with worse OS; conversely, higher M1 density demonstrated a correlation with OS improvement ${ }^{[85]}$.

In a retrospective study enrolling $68 \mathrm{MM}$ patients, an elevated CD68-positive and CD163-positive TAM expression showed a significant reduction of six-year OS, as confirmed by multivariate analysis. As a 
complementary finding, an increased CD163-positive M2 TAM number was associated with an elevated microvessel density ${ }^{[86]}$. In another relevant study, a reduced response was observed in patients presenting with high CD68-positive and CD163-positive TAM; however, only high CD163 TAM expression was related to a reduced PFS and OS. CD163 and CD168 were combined with ISS to design a new prognostic model $^{[87]}$. M2 TAM infiltration and correlation with pro-angiogenic factor CD147 were investigated in a spectrum from MGUS to recurrence MM. CD163 was used as the M2 marker and the cutoff for M2 infiltration was 100 per core. The authors showed a significant OS reduction in relapsed MM patients with high M2 expression (32 months vs. 6 months, $P=0.02$ ), suggesting a prognostic role of CD163-positive TAM in $\mathrm{MM}^{[88]}$. Finally, Andersen et al ${ }^{[8]}$ evaluated CD163 as a soluble marker in 104 blood samples and 17 BM samples in newly diagnosed MM patients. CD163 BM expression was higher compared to blood samples and was associated with a higher ISS stage. An elevated CD163 expression, with the suggested cutoff of $1.8 \mathrm{mg} / \mathrm{L}$, was associated with poor prognosis, further suggesting M2 TAM could favor MM growth and progression ${ }^{[89]}$.

\section{MINIMAL RESIDUAL DISEASE}

New drugs in MM have revolutionized the treatment paradigm and improved both progression-free and overall survival. However, progress in the definition of response is needed, since a CR has been defined for almost 15 years simply as the absence of a monoclonal component at immunofixation and a percentage of monoclonal plasma cells $<5 \%$ in the bone marrow.

The goal is to predict patients relapsing soon after the initial therapy and distinguish them from patients who maintain a long response (sustained CR) that is a surrogate for PFS and OS. Novel drugs are combined now in triplet or quadruplet treatment schedules and can implement in a high percentage of patients CR to a virtual disease disappearance. However, most MM patients still relapse. NGF and NGS have been introduced into clinical trials, bringing more sensitivity to detect minimal residual disease after therapy ${ }^{[23-34]}$. The detection limit of MRD is now considered $10^{-5}$; however, data suggest that a deeper limit of $10^{-6}$ or $10^{-7}$ could predict a better PFS ${ }^{[0]}$. Although NGS has been reported initially to have a deeper limit of detection $\left(10^{-6}\right)$ than NGF $\left(10^{-5}\right)$, both can now reach comparable sensitivity at $10^{-6}$.

\section{NGF and NGS in MM}

NGF has recently become part of the MRD evaluation, and it is based on the detection and quantification of normal PC vs. monoclonal PC using specific antibodies: PC markers such as CD38 and CD138; aberrant antigens expression such as $\mathrm{CD} 45^{\text {-low }}, \mathrm{CD} 19^{-}, \mathrm{CD} 27^{-}, \mathrm{CD} 81^{-}$, and $\mathrm{CD} 56$; CD28; CD117; and light chains $\kappa$ or $\kappa$. The Euro-Flow Consortium has introduced a more sensitive standardized technique based on two eightcolor tubes that permit detecting MRD with $100 \%$ applicability ${ }^{[24]}$ with a $4 \mathrm{~h}$ sample processing time. Sensitivity needs to be at least $10^{-5}$, but it has been reported as even better. A correct and adequate concentrate sample is needed to avoid hemodilution, and strategies to overcome CD38 monoclonal antibody interference have been described ${ }^{[26]}$.

NGS analyzes the clonal rearrangements of the immunoglobulin heavy chain (IgH) regions with parallel sequencing of reads with a sensitivity of $10^{-6}$. Importantly, patient primer construction is not needed, and the depth of detection is a strength ${ }^{[91]}$. Commercial kits are now available and FDA approved (LymphoSIGHT ${ }^{\oplus}$ and ImmunoSEQ, Adaptive Biotechnologies). Costs and a bioinformatic database for analysis as well as hemodilution are the limits ${ }^{[26]}$. NGF and NGS are recognized as complementary techniques, and the IMWG suggests utilizing the one available at the centers in clinical trials ${ }^{[36]}$. The Cassiopeia trial showed comparable sensitivity for NGF and NGS ${ }^{[13]}$. 


\section{Minimal residual disease sub-clone characterization}

MRD can be a surrogate for PFS and OS and is becoming an important tool for risk-stratification since the depth of response is correlated with prolonged survival ${ }^{[92]}$. In fact, a non-sustained CR can frequently be seen in MRD-positive MM patients. A goal in actual MM therapy could be to reach a sustained CR with MRD negativity lasting for at least one year. Searching for a sub-clone in MRD analysis could be an ideal way to identify drug resistance. It has been reported that MRD MM sub-clones could overexpress CD11a, CD11c, CD29, CD44, CD49d CD49e, CD54, CD138, CXCR4, and HLADR in the GEM2010MAS65 study involving 40 elderly patient ${ }^{[93]}$ treated with nine cycles of VMP (bortezomib, melphalan, and prednisone) or alternating VMP to Rd (lenalidomide and dexamethasone). In particular, integrins, chemokines, and adhesion molecules were overexpressed. These chemoresistant clones with a specific multi-flow signature also displayed genetic copy number alterations (CNA) that were present since diagnosis, and the resistant clone was selected after therapy resistance. The same group recently studied a larger number of patients $(n=$ 390 ) in the PETHEMA/GEM2012MENOS65 protocol (six induction cycles with Bor, Lena, and dexamethasone followed by autologous stem cell transplant, two consolidation cycles with Bor, Lena, and dexamethasone, and then randomization to maintenance therapy with Lena and dexamethasone $v s$. Ixa, Lena, and dexamethasone) ${ }^{[88]}$. They used NGF to identify detectable MRD and mechanisms related to resistance in 90 patients with high-risk (HR; i.e., del17p, t4;14, or t14;16) cytogenetics and 300 patients with standard-risk cytogenetics (SR; i.e., other anomalies not HR). Importantly, the results show the superiority of $90 \%$ PFS in MRD-negative patients vs. MRD-positive patients, irrespective of the cytogenetic status. Moreover, NGF studies and whole-genome sequencing showed clonal selection and higher genomic aberrations and mutations in 40 patients with multi-resistant clones ${ }^{[94]}$. Most mutations affected KRAS, $B R A F, C C N D 1, R O S 1, N R A S$, and FLT3 genes. CNA and mutations present at diagnosis were more likely to disappear in SR-cytogenetics patients, while HR patients had novel mutations during treatment, suggesting more genomic instability. In addition, when evaluating transcriptional clones, patients with HR cytogenetics showed the expression of PRDX6 and SOD1, which were related to a worst PFS. The authors concluded that, in HR cytogenetics MM patients, MRD resistance can arise from clones that evolve transcriptionally after therapy.

\section{Minimal residual disease negativity after current upfront therapies for transplant and non-transplant eligible myeloma patients}

With the introduction of novel drugs, responses can be seen in the majority of MM patients treated at diagnosis, both transplant and non-transplant eligible, with PFS lasting up to five years [Tables 2 and 3].

It is now evident that PFS alone cannot be the main parameter to judge new drugs in clinical trials. MRD has been proven to be a good prognosticator for PFS, OS, and drug resistance ${ }^{[2]}$. In the GIMEMA-MMY3006 study, VTD was compared to TD in 480 MM transplant eligible patients for induction therapy and double ASCT followed by consolidation therapy ${ }^{[95]}$. VTD incorporated into a double ASCT strategy plus consolidation therapy proved to be superior to TD in terms of CR attained ( $58 \% v s .41 \%$, respectively) and PFS and OS at 10 years ( $34 \%$ vs. $17 \%$ and $60 \%$ vs. $46 \%$, respectively) ${ }^{[96]}$. Although MRD was not done at that time, one study showed that, in a subset of patients treated with VTD consolidation after ASCT, MRD negativity was achieved and relapses were delayed ${ }^{[97]}$. In the IFM 2009, VRD was compared with VRD + ASCT, and in the latter group CR was superior ( $59 \%$ vs. $49 \%$ ) but OS did not differ significantly ${ }^{[98]}$. Importantly, MRD negativity was significantly correlated with improved three-year PFS ( $87 \% v s .42 \%)$, independently of the therapy received. Car was combined with Len and dexamethasone in the KRD regimen tested as induction and consolidation + maintenance therapy for up to $10 \mathrm{cycles}^{[93,99]}$. Although relatively few patients were treated, MRD negativity was reached in a high percentage of patients. Recently, Dara was added to VTD and compared to VTD alone in 1085 patients in the CASSIOPEIA study ${ }^{[13]}$ : response and MRD negativity were superior in Dara-VTD vs. VTD (64 \% vs. 44\%). Dara was added before 
Table 2. CR and MRD results in transplant eligible patients with currently available therapy

\begin{tabular}{|c|c|c|c|c|c|c|c|}
\hline Authors & Regimen & $n$ & $\begin{array}{l}\text { ASCT } \\
(\text { NO/1/2) }\end{array}$ & $\mathrm{C} / \mathrm{M}$ & CR (\%) & MRD neg (\%) & PFS/OS (months) \\
\hline Cavo et al..$^{[95]}$ & $\begin{array}{l}\text { TD } \\
\text { VTD }\end{array}$ & $\begin{array}{l}238 \\
236\end{array}$ & 2 & $C+M$ & $\begin{array}{l}41 \\
58\end{array}$ & NA & $\begin{array}{l}40.7 / 110 \\
59.6 / N R\end{array}$ \\
\hline Attal et al. ${ }^{[98]}$ & $\begin{array}{l}\text { VRD } \\
\text { VRD }\end{array}$ & $\begin{array}{l}350 \\
350\end{array}$ & $\begin{array}{l}\text { NO } \\
1\end{array}$ & $C+M$ & $\begin{array}{l}49 \\
59\end{array}$ & $\begin{array}{l}65 \\
80\end{array}$ & $\begin{array}{l}37 / \mathrm{NR} \\
50 / \mathrm{NR}\end{array}$ \\
\hline Jasielec et al. ${ }^{[99]}$ & KRD & 76 & 1 & $C+M$ & 78.9 & 70 & NR \\
\hline Moreau et al. ${ }^{[13]}$ & $\begin{array}{l}\text { Dara-VTD } \\
\text { VTD }\end{array}$ & $\begin{array}{l}543 \\
542\end{array}$ & 1 & $C+M$ & $\begin{array}{l}39 \\
26\end{array}$ & $\begin{array}{l}64 \\
44\end{array}$ & NR \\
\hline $\begin{array}{l}\text { Voorhees et al } .^{[100]} \\
\text { Laubach et al. }^{[101]}\end{array}$ & $\begin{array}{l}\text { Dara-VRD } \\
\text { VRD }\end{array}$ & $\begin{array}{l}103 \\
104\end{array}$ & 1 & $C+M$ & $\begin{array}{l}66 \\
47\end{array}$ & $\begin{array}{l}64 \\
30\end{array}$ & NR \\
\hline
\end{tabular}

PFS: Progression-free survival; OS: overall survival; CR: complete response; C + M: consolidation + maintenance; NA: not applicable; NR: not reached; ASCT: autologous stem cell transplantation; TD: thalidomide and dexamethasone; VTD: bortezomib, thalidomide, and dexamethasone; VRD: bortezomib, lenalidomide, and dexamethasone; KRD: carfilzomib, lenalidomide, and dexamethasone; Dara-VTD: daratumumab, bortezomib, thalidomide, and dexamethasone; Dara-VRD: daratumumab, bortezomib, lenalidomide, and dexamethasone.

Table 3. CR and MRD results in non-transplant eligible patients with currently available therapy

\begin{tabular}{llllll}
\hline Authors & Regimen & $\mathbf{n}$ & CR (\%) & MRD neg (\%) & PFS/OS (months) \\
\hline San Miguel et al. ${ }^{[102]}$ & VMP & 344 & 30 & NA & $24 / 56$ \\
& MP & 338 & 4 & & $16.6 / 43$ \\
Benboubker et al. ${ }^{[103]}$ & RD cont. & 535 & 15 & NA & $25.5 / 59$ \\
& RD18 & 541547 & 14 & & $20.7 / 62$ \\
& MPT & & 9 & & $21.2 / 49$ \\
Facon et al. ${ }^{[12]}$ & Dara-RD & 368 & 48 & 31 & NR \\
& RD & 369 & 25 & 10 & $34 / \mathrm{NR}$ \\
Mateos et al. & Dara-VMP & 356 & 46 & 28 & $36.4 / \mathrm{NR}$ \\
Durie et al. & VMP & 350 & 25 & 7 & $19.3 / \mathrm{NR}$ \\
& VRD & 235 & 24.2 & NA & $41 / \mathrm{NR}$ \\
& RD & 225 & 12.1 & & $29 / 69$ \\
\hline
\end{tabular}

PFS: Progression-free survival; OS: overall survival; CR: complete response.

ASCT to VRD in the GRIFFIN study and then compared to VRD alone ${ }^{[100,101]}$. Consolidation and maintenance therapy were also applied for up to 26 months of total therapy. MRD negativity was significantly superior in the Dara group (64\% vs. 30\%).

In the non-transplant eligible setting, great improvement with respect to the standard VMP or RD used until a few months ago ${ }^{[102-104]}$ was brought recently by the addition of daratumumab. The MAIA study ${ }^{[12]}$ compared Dara-Rd vs. Rd in MM patients not eligible for ASCT. At five years of follow-up, PFS has not been reached for the Dara-Rd group. CR was reached in $47 \%$ vs. $24 \%$ and MRD negativity was significantly higher $(31 \%$ vs. $10 \%)$. The Alcyone trial ${ }^{[11]}$ compared VMP vs. Dara-VMP. This study confirmed the superiority of the triplet with Dara, in both PFS and MRD negativity. Lastly, VRD was superior to RD in the SWOGS0777 trial in patients not proceeding to ASCT (PFS 41 months $v s .29$ months) ${ }^{[105]}$. Daratumumab was tested as consolidation therapy in patients achieving a > VGPR after ASCT in the DART4MM study ${ }^{[106,107]}$. An interim analysis showed MRD negativity in $45 \%$ of the patients at six months of treatment. Besides the great progress achieved with the new drugs with regard to the depth of responses, MRD status should not guide clinical decisions. 


\section{CONCLUSION}

MM patients still relapse after a variable period of remission. Drug resistance is a major cause of MM relapse with both intrinsic and extrinsic mechanisms. Therapeutic targeting of the bone marrow microenvironment and its interaction with MM plasma cells seems to be an ideal approach for the future. Besides the need for novel, more efficacious drugs, better prognosticators are also needed. MRD measured by NGF or NGS has entered into the MM diagnostic armamentarium and is a great prognosticator for novel drugs in clinical trials. Sustained MRD negativity is likely to be a long-term remission pre-requisite. Research has to be done to better clarify its role in identifying minimal residual sub-clones resistant to drugs commonly utilized in MM. NGF seems promising in this biological view. The characterization of these clones should be pursued in future large MM trials.

\section{DECLARATIONS}

\section{Authors' contributions}

Made substantial contributions to conception and design of the study: Gozzetti A, Pacelli P, Raspadori D, Tocci D

Wrote the manuscript: Gozzetti A, Ciofini S, Sicuranza A, Cencini E

Supervised the manuscript: Bocchia M

\section{Availability of data and materials}

Not applicable.

\section{Financial support and sponsorship}

None.

\section{Conflicts of interest}

All authors declared that there are no conflicts of interest.

\section{Ethical approval and consent to participate}

Not applicable.

\section{Consent for publication}

Not applicable.

\section{Copyright}

(c) The Author(s) 2022.

\section{REFERENCES}

1. Kumar SK, Rajkumar SV, Dispenzieri A, et al. Improved survival in multiple myeloma and the impact of novel therapies. Blood 2008;111:2516-20. DOI PubMed PMC

2. Brenner H, Gondos A, Pulte D. Recent major improvement in long-term survival of younger patients with multiple myeloma. Blood 2008;111:2521-6. DOI PubMed

3. Gozzetti A, Candi V, Papini G, Bocchia M. Therapeutic advancements in multiple myeloma. Front Oncol 2014;4:241. DOI PubMed PMC

4. Ocio EM, Richardson PG, Rajkumar SV, et al. New drugs and novel mechanisms of action in multiple myeloma in 2013: a report from the International Myeloma Working Group (IMWG). Leukemia 2014;28:525-42. DOI PubMed PMC

5. Gozzetti A, Cerase A. Novel agents in CNS myeloma treatment. Cent Nerv Syst Agents Med Chem 2014;14:23-7. DOI PubMed

6. Usmani SZ, Weiss BM, Plesner T, et al. Clinical efficacy of daratumumab monotherapy in patients with heavily pretreated relapsed or refractory multiple myeloma. Blood 2016;128:37-44. DOI PubMed

7. Jurczyszyn A, Olszewska-Szopa M, Hungria V, et al. Cutaneous involvement in multiple myeloma: a multi-institutional retrospective study of 53 patients. Leuk Lymphoma 2016;57:2071-6. DOI PubMed

8. Kocoglu MH, Badros AZ. Newly diagnosed multiple myeloma: current treatment strategies, emerging therapeutic approaches and beyond. Expert Rev Hematol 2020;13:669-86. DOI PubMed 
9. Chari A, Suvannasankha A, Fay JW, et al. Daratumumab plus pomalidomide and dexamethasone in relapsed and/or refractory multiple myeloma. Blood 2017;130:974-81. DOI PubMed

10. Dimopoulos MA, Terpos E, Boccadoro M, et al. Daratumumab plus pomalidomide and dexamethasone versus pomalidomide and dexamethasone alone in previously treated multiple myeloma (APOLLO): an open-label, randomised, phase 3 trial. Lancet Oncol 2021;22:801-12. DOI PubMed

11. Mateos M, Cavo M, Blade J, et al. Overall survival with daratumumab, bortezomib, melphalan, and prednisone in newly diagnosed multiple myeloma (ALCYONE): a randomised, open-label, phase 3 trial. Lancet 2020;395:132-41. DOI PubMed

12. Facon T, Kumar S, Plesner T, et al; MAIA Trial Investigators. Daratumumab plus lenalidomide and dexamethasone for untreated myeloma. N Engl J Med 2019;380:2104-15. DOI PubMed

13. Moreau P, Attal M, Hulin C, et al. Bortezomib, thalidomide, and dexamethasone with or without daratumumab before and after autologous stem-cell transplantation for newly diagnosed multiple myeloma (CASSIOPEIA): a randomised, open-label, phase 3 study. Lancet 2019;394:29-38. DOI PubMed

14. Mohty M, Terpos E, Mateos MV, et al; EMMOS Investigators. Multiple myeloma treatment in real-world clinical practice: results of a prospective, multinational, noninterventional study. Clin Lymphoma Myeloma Leuk 2018;18:e401-19. DOI PubMed

15. Gozzetti A, Cerase A, Lotti F, et al; GIMEMA (Gruppo Italiano Malattie Ematologiche dell'Adulto) Myeloma Working Party. Extramedullary intracranial localization of multiple myeloma and treatment with novel agents: a retrospective survey of 50 patients. Cancer 2012;118:1574-84. DOI PubMed

16. Castillo JJ, Jurczyszyn A, Brozova L, et al. IgM myeloma: a multicenter retrospective study of 134 patients. Am J Hematol 2017;92:746-51. DOI PubMed

17. Jurczyszyn A, Radocha J, Davila J, et al. Prognostic indicators in primary plasma cell leukaemia: a multicentre retrospective study of 117 patients. Br J Haematol 2018;180:831-9. DOI PubMed

18. Rajan AM, Rajkumar SV. Interpretation of cytogenetic results in multiple myeloma for clinical practice. Blood Cancer $J$ 2015;5:e365. DOI PubMed PMC

19. Gozzetti A, Le Beau MM. Fluorescence in situ hybridization: uses and limitations. Semin Hematol 2000;37:320-33. DOI PubMed

20. Goldman-Mazur S, Jurczyszyn A, Castillo JJ, et al. A multicenter retrospective study of 223 patients with $t(14 ; 16)$ in multiple myeloma. Am J Hematol 2020;95:503-9. DOI PubMed

21. Keats JJ, Chesi M, Egan JB, et al. Clonal competition with alternating dominance in multiple myeloma. Blood 2012;120:1067-76. DOI PubMed PMC

22. Davis LN, Sherbenou DW. Emerging therapeutic strategies to overcome drug resistance in multiple myeloma. Cancers (Basel) 2021;13:1686. DOI PubMed PMC

23. Paiva B, van Dongen JJ, Orfao A. New criteria for response assessment: role of minimal residual disease in multiple myeloma. Blood 2015;125:3059-68. DOI PubMed PMC

24. Flores-Montero J, Sanoja-Flores L, Paiva B, et al. Next Generation Flow for highly sensitive and standardized detection of minimal residual disease in multiple myeloma. Leukemia 2017;31:2094-103. DOI PubMed PMC

25. Romano A, Palumbo GA, Parrinello NL, Conticello C, Martello M, Terragna C. Minimal residual disease assessment within the bone marrow of multiple myeloma: a review of caveats, clinical significance and future perspectives. Front Oncol 2019;9:699. DOI PubMed PMC

26. Gozzetti A, Raspadori D, Bacchiarri F, et al. Minimal residual disease in multiple myeloma: state of the art and applications in clinical practice. J Pers Med 2020;10:120. DOI PubMed PMC

27. Rawstron AC, Orfao A, Beksac M, et al; European Myeloma Network. Report of the European Myeloma Network on multiparametric flow cytometry in multiple myeloma and related disorders. Haematologica 2008;93:431-8. DOI PubMed

28. Brooimans RA, Kraan J, van Putten W, Cornelissen JJ, Löwenberg B, Gratama JW. Flow cytometric differential of leukocyte populations in normal bone marrow: influence of peripheral blood contamination. Cytometry B Clin Cytom 2009;76:18-26. DOI PubMed

29. Gupta R, Bhaskar A, Kumar L, Sharma A, Jain P. Flow cytometric immunophenotyping and minimal residual disease analysis in multiple myeloma. Am J Clin Pathol 2009;132:728-32. DOI PubMed

30. Delgado JA, Guillén-Grima F, Moreno C, et al. A simple flow-cytometry method to evaluate peripheral blood contamination of bone marrow aspirates. J Immunol Methods 2017;442:54-8. DOI PubMed

31. Loken MR, Chu SC, Fritschle W, Kalnoski M, Wells DA. Normalization of bone marrow aspirates for hemodilution in flow cytometric analyses. Cytometry B Clin Cytom 2009;76:27-36. DOI PubMed

32. Yanamandra U, Kumar SK. Minimal residual disease analysis in myeloma - when, why and where. Leuk Lymphoma 2018;59:177284. DOI PubMed PMC

33. Bai Y, Orfao A, Chim CS. Molecular detection of minimal residual disease in multiple myeloma. Br J Haematol 2018;181:11-26. DOI PubMed

34. Yao Q, Bai Y, Orfao A, Chim CS. Standardized minimal residual disease detection by next-generation sequencing in multiple myeloma. Front Oncol 2019;9:449. DOI PubMed PMC

35. Perrot A, Lauwers-Cances V, Corre J, et al. Minimal residual disease negativity using deep sequencing is a major prognostic factor in multiple myeloma. Blood 2018;132:2456-64. DOI PubMed PMC

36. Kumar S, Paiva B, Anderson KC, et al. International Myeloma Working Group consensus criteria for response and minimal residual disease assessment in multiple myeloma. Lancet Oncol 2016;17:e328-46. DOI PubMed

37. Munshi NC, Avet-Loiseau H, Anderson KC, et al. A large meta-analysis establishes the role of MRD negativity in long-term survival 
outcomes in patients with multiple myeloma. Blood Adv 2020;4:5988-99. DOI PubMed PMC

38. Berger N, Kim-Schulze S, Parekh S. Minimal residual disease in multiple myeloma: impact on response assessment, prognosis and tumor heterogeneity. Adv Exp Med Biol 2018;1100:141-59. DOI PubMed

39. Chauhan D, Hideshima T, Mitsiades C, et al. Proteasome inhibitor therapy in multiple myeloma. Mol Cancer Ther 2005;4:686-92. DOI PubMed

40. Gozzetti A, Papini G, Candi V, Brambilla CZ, Sirianni S, Bocchia M. Second generation proteasome inhibitors in multiple myeloma. Anticancer Agents Med Chem 2017;17:920-6. DOI PubMed

41. Ciechanover A. The ubiquitin-proteasome proteolytic pathway. Cell 1994;79:13-21. DOI PubMed

42. Barrio S, Stühmer T, Da-Viá M, et al. Spectrum and functional validation of PSMB5 mutations in multiple myeloma. Leukemia 2019;33:447-56. DOI PubMed

43. Robak P, Drozdz I, Szemraj J, Robak T. Drug resistance in multiple myeloma. Cancer Treat Rev 2018;70:199-208. DOI PubMed

44. Leung-Hagesteijn C, Erdmann N, Cheung G, et al. Xbp1s-negative tumor B cells and pre-plasmablasts mediate therapeutic proteasome inhibitor resistance in multiple myeloma. Cancer Cell 2013;24:289-304. DOI PubMed PMC

45. Abraham J, Salama NN, Azab AK. The role of P-glycoprotein in drug resistance in multiple myeloma. Leuk Lymphoma 2015;56:2633. DOI PubMed

46. Besse A, Stolze SC, Rasche L, et al. Carfilzomib resistance due to ABCB1/MDR1 overexpression is overcome by nelfinavir and lopinavir in multiple myeloma. Leukemia 2018;32:391-401. DOI PubMed PMC

47. O'Connor R, Ooi MG, Meiller J, et al. The interaction of bortezomib with multidrug transporters: implications for therapeutic applications in advanced multiple myeloma and other neoplasias. Cancer Chemother Pharmacol 2013;71:1357-68. DOI PubMed PMC

48. Kubiczkova L, Pour L, Sedlarikova L, Hajek R, Sevcikova S. Proteasome inhibitors - molecular basis and current perspectives in multiple myeloma. J Cell Mol Med 2014;18:947-61. DOI PubMed PMC

49. Baranowska K, Misund K, Starheim KK, et al. Hydroxychloroquine potentiates carfilzomib toxicity towards myeloma cells. Oncotarget 2016;7:70845-56. DOI PubMed PMC

50. Xu H, Han H, Song S, et al. Exosome-transmitted PSMA3 and PSMA3-AS1 promote proteasome inhibitor resistance in multiple myeloma. Clin Cancer Res 2019;25:1923-35. DOI PubMed

51. Mitsiades CS, Mitsiades NS, McMullan CJ, et al. Antimyeloma activity of heat shock protein-90 inhibition. Blood 2006;107:1092100. DOI PubMed PMC

52. Kuhn DJ, Berkova Z, Jones RJ, et al. Targeting the insulin-like growth factor-1 receptor to overcome bortezomib resistance in preclinical models of multiple myeloma. Blood 2012;120:3260-70. DOI PubMed PMC

53. Stessman HA, Baughn LB, Sarver A, et al. Profiling bortezomib resistance identifies secondary therapies in a mouse myeloma model. Mol Cancer Ther 2013;12:1140-50. DOI PubMed PMC

54. Richardson P, Hideshima T, Anderson K. Thalidomide in multiple myeloma. Biomed Pharmacother 2002;56:115-28. DOI PubMed

55. Lopez-Girona A, Mendy D, Ito T, et al. Cereblon is a direct protein target for immunomodulatory and antiproliferative activities of lenalidomide and pomalidomide. Leukemia 2012;26:2326-35. DOI PubMed PMC

56. Chamberlain PP, Lopez-Girona A, Miller K, et al. Structure of the human Cereblon-DDB1-lenalidomide complex reveals basis for responsiveness to thalidomide analogs. Nat Struct Mol Biol 2014;21:803-9. DOI PubMed

57. Hu S, Yuan L, Yan H, Li Z. Design, synthesis and biological evaluation of Lenalidomide derivatives as tumor angiogenesis inhibitor. Bioorg Med Chem Lett 2017;27:4075-81. DOI PubMed

58. Kortüm KM, Mai EK, Hanafiah NH, et al. Targeted sequencing of refractory myeloma reveals a high incidence of mutations in CRBN and Ras pathway genes. Blood 2016;128:1226-33. DOI PubMed PMC

59. Bertrand E, Jouy N, Manier S, et al. Role of IRF4 in resistance to immunomodulatory (IMid) compounds ${ }^{\circledR}$ in Waldenström's macroglobulinemia. Oncotarget 2017;8:112917-27. DOI PubMed PMC

60. Haertle L, Barrio S, Munawar U, et al. Cereblon enhancer methylation and IMiD resistance in multiple myeloma. Blood 2021;138:1721-6. DOI PubMed PMC

61. Lokhorst HM, Plesner T, Laubach JP, et al. Targeting CD38 with daratumumab monotherapy in multiple myeloma. $N$ Engl J Med 2015;373:1207-19. DOI PubMed

62. Dimopoulos MA, Oriol A, Nahi H, et al; POLLUX Investigators. Daratumumab, lenalidomide, and dexamethasone for multiple myeloma. N Engl J Med 2016;375:1319-31. DOI PubMed

63. Palumbo A, Chanan-Khan A, Weisel K, et al; CASTOR Investigators. Daratumumab, bortezomib, and dexamethasone for multiple myeloma. N Engl J Med 2016;375:754-66. DOI PubMed

64. Lonial S, Dimopoulos M, Palumbo A, et al; ELOQUENT-2 Investigators. Elotuzumab therapy for relapsed or refractory multiple myeloma. N Engl J Med 2015;373:621-31. DOI PubMed

65. Dimopoulos M, Quach H, Mateos M, et al. Carfilzomib, dexamethasone, and daratumumab versus carfilzomib and dexamethasone for patients with relapsed or refractory multiple myeloma (CANDOR): results from a randomised, multicentre, open-label, phase 3 study. Lancet 2020;396:186-97. DOI PubMed

66. Nijhof IS, Casneuf T, van Velzen J, et al. CD38 expression and complement inhibitors affect response and resistance to daratumumab therapy in myeloma. Blood 2016;128:959-70. DOI PubMed

67. Varga C, Maglio M, Ghobrial IM, Richardson PG. Current use of monoclonal antibodies in the treatment of multiple myeloma. $\mathrm{Br} J$ Haematol 2018;181:447-59. DOI PubMed

68. de Weers M, Tai YT, van der Veer MS, et al. Daratumumab, a novel therapeutic human CD38 monoclonal antibody, induces killing 
of multiple myeloma and other hematological tumors. J Immunol 2011;186:1840-8. DOI PubMed

69. Overdijk MB, Verploegen S, Bögels M, et al. Antibody-mediated phagocytosis contributes to the anti-tumor activity of the therapeutic antibody daratumumab in lymphoma and multiple myeloma. MAbs 2015;7:311-21. DOI PubMed PMC

70. Pick M, Vainstein V, Goldschmidt N, et al. Daratumumab resistance is frequent in advanced-stage multiple myeloma patients irrespective of CD38 expression and is related to dismal prognosis. Eur J Haematol 2018;100:494-501. DOI PubMed

71. Cho SF, Lin L, Xing L, et al. BCMA-targeting therapy: driving a new era of immunotherapy in multiple myeloma. Cancers (Basel) 2020;12:1473. DOI PubMed PMC

72. Nijhof IS, Groen RW, Lokhorst HM, et al. Upregulation of CD38 expression on multiple myeloma cells by all-trans retinoic acid improves the efficacy of daratumumab. Leukemia 2015;29:2039-49. DOI PubMed

73. Gupta VA, Matulis SM, Conage-Pough JE, et al. Bone marrow microenvironment-derived signals induce Mcl-1 dependence in multiple myeloma. Blood 2017;129:1969-79. DOI PubMed PMC

74. Ria R, Vacca A. Bone marrow stromal cells-induced drug resistance in multiple myeloma. Int J Mol Sci 2020;21:613. DOI PubMed PMC

75. Cencini E, Fabbri A, Sicuranza A, Gozzetti A, Bocchia M. The role of tumor-associated macrophages in hematologic malignancies. Cancers (Basel) 2021;13:3597. DOI PubMed PMC

76. Beider K, Bitner H, Leiba M, et al. Multiple myeloma cells recruit tumor-supportive macrophages through the CXCR4/CXCL12 axis and promote their polarization toward the M2 phenotype. Oncotarget 2014;5:11283-96. DOI PubMed PMC

77. Zheng Y, Cai Z, Wang S, et al. Macrophages are an abundant component of myeloma microenvironment and protect myeloma cells from chemotherapy drug-induced apoptosis. Blood 2009;114:3625-8. DOI PubMed PMC

78. Zheng Y, Yang J, Qian J, et al. PSGL-1/selectin and ICAM-1/CD18 interactions are involved in macrophage-induced drug resistance in myeloma. Leukemia 2013;27:702-10. DOI PubMed PMC

79. Kim J, Denu RA, Dollar BA, et al. Macrophages and mesenchymal stromal cells support survival and proliferation of multiple myeloma cells. Br J Haematol 2012;158:336-46. DOI PubMed PMC

80. Scavelli C, Nico B, Cirulli T, et al. Vasculogenic mimicry by bone marrow macrophages in patients with multiple myeloma. Oncogene 2008;27:663-74. DOI PubMed

81. Ribatti D, Vacca A. The role of monocytes-macrophages in vasculogenesis in multiple myeloma. Leukemia 2009;23:1535-6. DOI PubMed

82. De Beule N, De Veirman K, Maes K, et al. Tumour-associated macrophage-mediated survival of myeloma cells through STAT3 activation. J Pathol 2017;241:534-46. DOI PubMed

83. Cencini E, Fabbri A, Bocchia M. Prognostic role of M2 tumour-associated macrophages in lymphoproliferative disorders. $J$ Pathol 2017;242:511-2. DOI PubMed

84. Wang H, Hu WM, Xia ZJ, et al. High numbers of CD163+ tumor-associated macrophages correlate with poor prognosis in multiple myeloma patients receiving bortezomib-based regimens. J Cancer 2019;10:3239-45. DOI PubMed PMC

85. Andersen MN, Andersen NF, Rødgaard-Hansen S, Hokland M, Abildgaard N, Møller HJ. The novel biomarker of alternative macrophage activation, soluble mannose receptor (sMR/sCD206): implications in multiple myeloma. Leuk Res 2015;39:971-5. DOI PubMed

86. Suyanı E, Sucak GT, Akyürek N, et al. Tumor-associated macrophages as a prognostic parameter in multiple myeloma. Ann Hematol 2013;92:669-77. DOI PubMed

87. Chen X, Chen J, Zhang W, et al. Prognostic value of diametrically polarized tumor-associated macrophages in multiple myeloma. Oncotarget 2017;8:112685-96. DOI PubMed PMC

88. Panchabhai S, Kelemen K, Ahmann G, Sebastian S, Mantei J, Fonseca R. Tumor-associated macrophages and extracellular matrix metalloproteinase inducer in prognosis of multiple myeloma. Leukemia 2016;30:951-4. DOI PubMed

89. Andersen MN, Abildgaard N, Maniecki MB, Møller HJ, Andersen NF. Monocyte/macrophage-derived soluble CD163: a novel biomarker in multiple myeloma. Eur J Haematol 2014;93:41-7. DOI PubMed

90. Landgren O, Rajkumar SV. New developments in diagnosis, prognosis, and assessment of response in multiple myeloma. Clin Cancer Res 2016;22:5428-33. DOI PubMed PMC

91. Lionetti M, Neri A. Utilizing next-generation sequencing in the management of multiple myeloma. Expert Rev Mol Diagn 2017;17:653-63. DOI PubMed

92. Munshi NC, Avet-Loiseau H, Rawstron AC, et al. Association of minimal residual disease with superior survival outcomes in patients with multiple myeloma: a meta-analysis. JAMA Oncol 2017;3:28-35. DOI PubMed PMC

93. Paiva B, Corchete LA, Vidriales MB, et al; Spanish Myeloma Group/Program for the Study of Malignant Blood Diseases Therapeutics (GEM / PETHEMA) Cooperative Study Groups. Phenotypic and genomic analysis of multiple myeloma minimal residual disease tumor cells: a new model to understand chemoresistance. Blood 2016;127:1896-906. DOI PubMed

94. Goicoechea I, Puig N, Cedena MT, et al. Deep MRD profiling defines outcome and unveils different modes of treatment resistance in standard- and high-risk myeloma. Blood 2021;137:49-60. DOI PubMed

95. Cavo M, Tacchetti P, Patriarca F, et al. Bortezomib with thalidomide plus dexamethasone compared with thalidomide plus dexamethasone as induction therapy before, and consolidation therapy after, double autologous stem-cell transplantation in newly diagnosed multiple myeloma: a randomised phase 3 study. Lancet 2010;376:2075-85. DOI PubMed

96. Tacchetti P, Pantani L, Patriarca F, et al. Bortezomib, thalidomide, and dexamethasone followed by double autologous haematopoietic stem-cell transplantation for newly diagnosed multiple myeloma (GIMEMA-MMY-3006): long-term follow-up analysis of a randomised phase 3, open-label study. Lancet Haematology 2020;7:e861-73. DOI PubMed 
97. Ladetto M, Pagliano G, Ferrero S, et al. Major tumor shrinking and persistent molecular remissions after consolidation with bortezomib, thalidomide, and dexamethasone in patients with autografted myeloma. J Clin Oncol 2010;28:2077-84. DOI PubMed

98. Attal M, Lauwers-Cances V, Hulin C, et al; IFM 2009 Study. Lenalidomide, bortezomib, and dexamethasone with transplantation for myeloma. N Engl J Med 2017;376:1311-20. DOI PubMed

99. Jasielec JK, Kubicki T, Raje N, et al. Carfilzomib, lenalidomide, and dexamethasone plus transplant in newly diagnosed multiple myeloma. Blood 2020;136:2513-23. DOI PubMed PMC

100. Voorhees PM, Rodriguez C, Reeves B, et al. Daratumumab plus RVd for newly diagnosed multiple myeloma: final analysis of the safety run-in cohort of GRIFFIN. Blood Adv 2021;5:1092-6. DOI PubMed PMC

101. Laubach JP, Kaufman JL, Sborov DW, et al. Daratumumab (DARA) plus lenalidomide, bortezomib, and dexamethasone (RVd) in patients (Pts) with transplant-eligible newly diagnosed multiple myeloma (NDMM): updated analysis of griffin after 24 months of maintenance. Blood 2021;138:79. DOI

102. San Miguel JF, Schlag R, Khuageva NK, et al; VISTA Trial Investigators. Bortezomib plus melphalan and prednisone for initial treatment of multiple myeloma. N Engl J Med 2008;359:906-17. DOI PubMed

103. Benboubker L, Dimopoulos MA, Dispenzieri A, et al; FIRST Trial Team. Lenalidomide and dexamethasone in transplant-ineligible patients with myeloma. N Engl J Med 2014;371:906-17. DOI PubMed

104. Facon T, Dimopoulos MA, Dispenzieri A, et al. Final analysis of survival outcomes in the phase 3 FIRST trial of up-front treatment for multiple myeloma. Blood 2018;131:301-10. DOI PubMed PMC

105. Durie BGM, Hoering A, Sexton R, et al. Longer term follow-up of the randomized phase III trial SWOG S0777: bortezomib, lenalidomide and dexamethasone vs. lenalidomide and dexamethasone in patients (Pts) with previously untreated multiple myeloma without an intent for immediate autologous stem cell transplant (ASCT). Blood Cancer J 2020;10:53. DOI PubMed PMC

106. Gozzetti A, Raspadori D, Bacchiarri F, et al. DART4MM: daratumumab as consolidation therapy in patients who already achieved optimal response/MRD positivity by next generation flow (NGF): preliminary results of a phase 2 multicenter study. Clin Lymphoma Myeloma Leuk 2019;19:e161-2. DOI

107. Gozzetti A, Bacchiarri F, Sammartano V, et al. Long-term safety of rapid daratumumab infusions in multiple myeloma patients. Front Oncol 2020;10:570187. DOI PubMed PMC 